\title{
Staphylococcal Exotoxins Induce Interleukin 22 in Human Th22 Cells
}

\author{
Margarete Niebuhr Julia Mainardy Annice Heratizadeh \\ Imke Satzger Thomas Werfel \\ Department of Dermatology and Allergy, Division of Immunodermatology and Allergy Research, \\ Hannover Medical School, Hannover, Germany
}

\section{Key Words \\ Staphylococcus aureus - Staphylococcal enterotoxin B . a-Toxin $\cdot$ IL-22 $\cdot$ T helper 22 cells $\cdot$ Human memory T cells}

\begin{abstract}
Background: We have shown previously that $T$ cells from atopic dermatitis (AD) patients produce more IL-22 upon staphylococcal exotoxin stimulation compared to psoriasis patients and healthy controls. The role of staphylococcal exotoxins on polarized memory $T$ helper (Th) 22 cells which are enriched in inflamed AD skin remains elusive. Our aim was to investigate IL-22 production in response to staphylococcal enterotoxin B (SEB) and a-toxin stimulation in human memory T cells and polarized Th22 cells. Methods: IL-22 induction was investigated in human peripheral blood-derived CD4+CD45RO+CD45RA- T cells and polarized Th22 cells after SEB and sublytic a-toxin stimulation in a time-dependent manner at the mRNA and protein (ELISA) levels. Results: Th22 cells secreted more IL-22 compared to freshly isolated peripheral blood-derived memory $T$ cells. SEB and a-toxin induced IL-22 in memory T cells as well as in Th22 cells. More IL-22 was induced by SEB and a-toxin in freshly isolated peripheral blood memory T cells compared to Th22 cells derived from memory $T$ cells in long-term cell culture without polarization and Th22 cells under Th22-promoting conditions with IL-6 and TNF-a. No differences in IL-22 induction by staphylococcal exotoxins were observed between
\end{abstract}

\section{KARGER}

E-Mail karger@karger.com

www.karger.com/iaa cells from $A D$ compared to psoriasis patients and healthy controls. Conclusions: Increased IL-22 secretion can promptly be induced by staphylococcal exotoxins in skin infiltrating CD4+CD45RO+CD45RA- memory T cells and can potentially amplify chronic skin inflammation in $A D$ in the context of bacterial colonization and infection. This should be investigated further in detail in lesional skin of $A D$ and psoriasis patients.

(c) 2014 S. Karger AG, Basel

\section{Introduction}

Some years ago a distinct subset of IL-22-producing CD4+ T cells, namely T helper $(\mathrm{Th}) 22$ cells, were characterized as IL-22-producing T cells different from Th1, Th2 and Th17 cells. These cells concomitantly express the chemokine receptor CCR6 and the skin-homing receptors CCR4 and CCR10 and produce high levels of IL-22, but do not express IL-17 or IFN- $\gamma$. Additionally, Th22 cells were found to produce high levels of IL-13 and TNF- $\alpha[1-3]$.

We demonstrated in a previous study that the staphylococcal exotoxins, staphylococcal exotoxin B (SEB) and a-toxin, were strong inducers of IL-22 in CD4+ T cells. $\mathrm{CD} 4+\mathrm{T}$ cells from atopic dermatitis (AD) patients secreted significantly more IL-22 compared to psoriasis patients and healthy controls [4]. The aim of this study was to fur- 
Table 1. Characterization of human memory $\mathrm{T}$ cells and Th22 cells

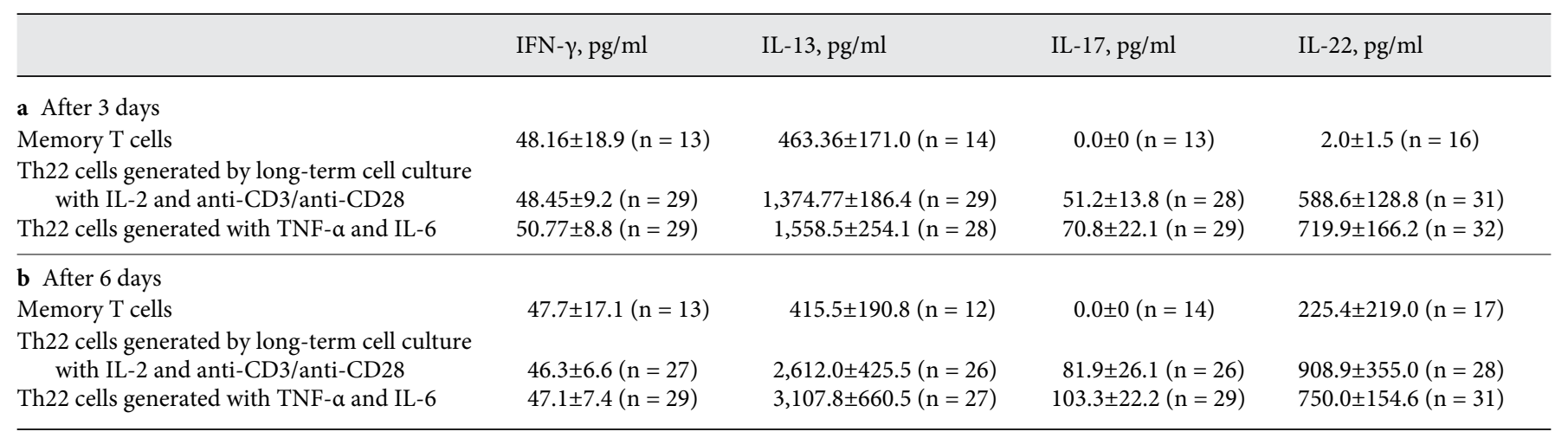

Data are shown as mean values \pm SEM after 3 or 6 days of cell culture. Th1 (IFN- $\gamma$ ), Th2 (IL-13), Th17 (IL-17) and Th22 (IL-22) cytokine secretion in human CD4+ memory T cells, Th22 cells generated by long-term cell culture with IL- 2 and anti-CD3/anti-CD28 and Th22 cells generated with TNF- $\alpha$ and IL-6. Cell-free culture supernatants were quantified for IFN- $\gamma$, IL-13, IL-17, and IL-22 secretion by ELISA.

ther investigate IL-22 secretion upon stimulation with staphylococcal exotoxins in human peripheral blood-derived CD4+CD45RO+CD45RA- memory T cells and polarized Th22 cells since highly activated memory $\mathrm{T}$ cells are enriched in inflamed AD skin, favor a tissue phenotype and are distinct from freshly isolated CD4+ T cells.

\section{Material and Methods}

\section{Patients}

Peripheral blood samples were taken from healthy donors and patients with exacerbated psoriasis $(n=9)$ and $\mathrm{AD}(\mathrm{n}=8)$, who presented to our outpatient and inpatient department as previously described [5]. AD was determined by the diagnostic criteria of Hanifin and Rajka [6]. The study was approved by the local ethics committee (No. 5627) and was in accordance with the protocols of the Declaration of Helsinki. All subjects gave informed consent.

Further information about patients and methods is given in the online supplementary material (for all online suppl. material, see www.karger.com/doi/10.1159/000367923).

\section{Results}

\section{Characterization of Human Memory T Cells and}

Th22 Cells

Th22 cells from CD4+CD45RO+CD45RA- memory $T$ cells cultivated in the presence of IL- 6 and TNF- $\alpha$ secreted more IL-22 compared to Th22 cells generated by long-term cell culture with IL-2 and anti-CD3/anti-CD28 or freshly isolated peripheral blood memory $\mathrm{T}$ cells after 3 days of cell culture as assessed by ELISA (table 1a). After 6 days of cell culture Th22 cells generated with IL-2 and
anti-CD3/anti-CD28 had an enhanced capacity to produce IL-22 compared to Th22 cells generated in an IL-6and TNF- $\alpha$-dependent manner and freshly isolated memory T cells (table $1 \mathrm{~b}$ ). Th22 cells coexpressed IL-13 and are therefore considered as Th22/Th2 cells (table 1).

Intracellular flow cytometric analysis revealed an increase in IL-22 production in Th22 cells generated by long-term cell culture with IL-2 and anti-CD3/anti-CD28 (5.5\%) and in Th22 cells cultivated in the presence of IL-6 and TNF- $\alpha(5.7 \%)$ compared to freshly isolated memory T cells ( $0.2 \%$; online suppl. fig. S1).

\section{Induction of IL-22 by SEB and $\alpha$-Toxin in Human}

CD4+ Memory and Th22 Cells at the mRNA Level

IL-22 expression after SEB and $\alpha$-toxin stimulation was investigated at the mRNA level by quantitative RT-PCR.

CD4+CD45RO+CD45RA - memory T cells and Th22 cells were either left unstimulated or stimulated with SEB or a-toxin, respectively, for 4 and $8 \mathrm{~h}$. Stimulation with a-toxin or SEB yielded a significant upregulation of IL-22 mRNA in freshly isolated peripheral blood memory $\mathrm{T}$ cells (fig. 1a), as well as in Th22 cells derived from memory $\mathrm{T}$ cells in long-term cell culture without Th22-promoting conditions (fig. 1b) and Th22 cells cultured with TNF- $\alpha$ plus IL-6 (fig. 1c).

SEB induced a mean 7,095-fold increase in IL-22 mRNA expression in freshly isolated peripheral blood memory T cells (fig. 1a), whereas a median 356- and 334fold increase in IL-22 mRNA expression was induced after $4 \mathrm{~h}$ of SEB stimulation in Th22 cells derived from memory $\mathrm{T}$ cells in long-term cell culture without polarization (fig. 1b) and Th22 cells generated with IL-6 and TNF- $\alpha$ DOI: $10.1159 / 000367923$
Niebuhr/Mainardy/Heratizadeh/Satzger/ Werfel 


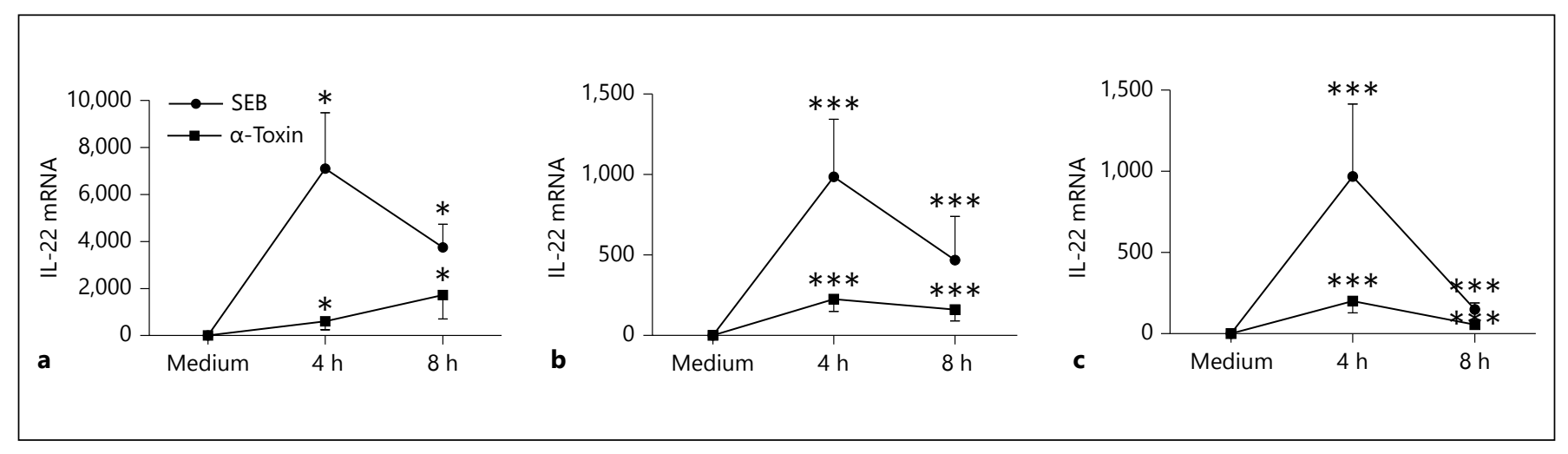

Fig. 1. IL-22 is induced after 4 and $8 \mathrm{~h}$ of SEB $(100 \mathrm{ng} / \mathrm{ml})$ and a-toxin $(50 \mathrm{ng} / \mathrm{ml})$ stimulation in freshly isolated peripheral blood memory T cells $(\mathbf{a} ; \mathrm{n}=4-5)$ as well as in Th22 cells derived from memory $\mathrm{T}$ cells in long-term cell culture without polarization (b; $\mathrm{n}=20-21)$ and Th22 cells polarized with IL-6 and TNF- $\alpha$ (c; $\mathrm{n}=21$ ) at the mRNA level (normalized ratio). Data are shown as mean IL-22/GAPDH ratio + SEM. ${ }^{*} \mathrm{p}<0.05$; $^{* *} \mathrm{p}<0.001$.

upon 72 and $144 \mathrm{~h}$ of SEB and $\alpha$-toxin stimulation than the appropriate medium control. SEB and $\alpha$-toxin stimulation for $144 \mathrm{~h}$ did not enhance IL-22 secretion compared to stimulation of $72 \mathrm{~h}$. SEB and $a$-toxin had the highest capacity of IL-22 induction in freshly isolated peripheral blood memory T cells (fig. $2 \mathrm{a}$ ) compared to Th22 cells derived from memory $\mathrm{T}$ cells in long-term cell culture without Th22-promoting conditions (fig. 2b) and Th22 cells generated with IL- 6 and TNF- $\alpha$ (fig. $2 c$ ). No differences in IL-22 secretion upon stimulation with staphylococcal exotoxins were observed between cells from $\mathrm{AD}$ patients and cells from psoriasis patients and healthy controls (data not shown).

\section{Discussion}

In a previous study we demonstrated that SEB and sublytic concentrations of $\alpha$-toxin are strong inducers of IL-22 in peripheral blood mononuclear cells (PBMCs), T cells and autologous cocultures of keratinocytes and CD4+ T cells. Moreover, we showed an enhanced IL-22 secretion by PBMCs and CD4+ T cells obtained from AD patients compared to psoriasis patients and healthy controls upon stimulation with $\alpha$-toxin. This observation was significant in $\mathrm{T}$ cells from $\mathrm{AD}$ patients compared to psoriasis patients and healthy controls [4]. As evidence has increased that Th22 cells play an important role in chronic inflammatory skin disorders, namely AD and psoriasis, we investigated in the present study whether the staphylococcal exotoxins SEB and $\alpha$-toxin were sufficient to stimulate IL-22 secretion in polarized peripheral blood-derived memory Th22 

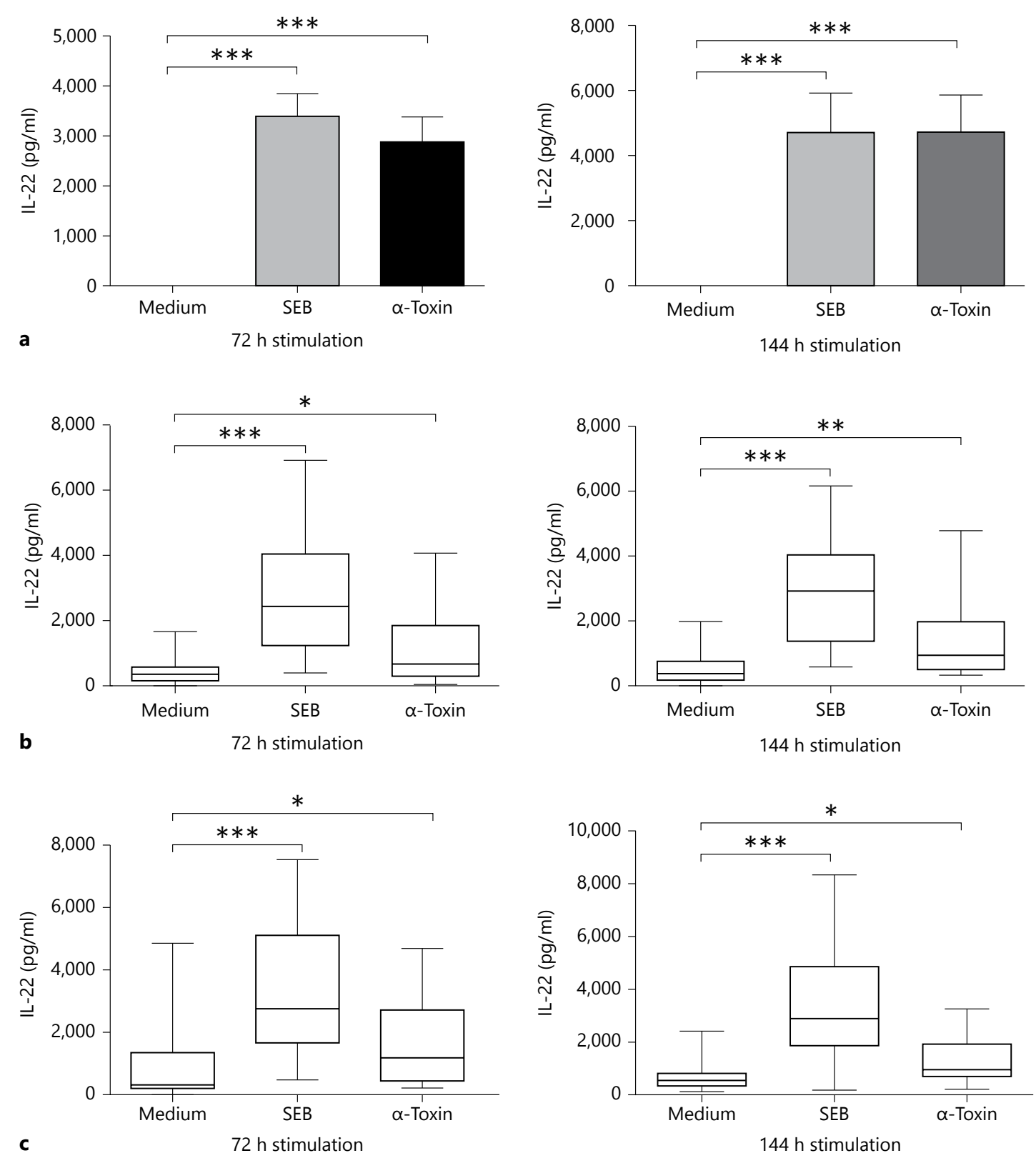

Fig. 2. Increased IL-22 secretion in freshly isolated peripheral blood memory T cells (a; $\mathrm{n}=14$ left, $\mathrm{n}=13$ right) in Th22 cells derived from memory $\mathrm{T}$ cells in long-term cell culture $(\mathbf{b} ; \mathrm{n}=$ 21-24 left, $\mathrm{n}=18$ right) and Th22 cells generated with IL-6 and TNF- $\alpha$ (c; $n=22-23$ left, $n=18-20$ right) induced by staphylo-

cells since highly activated memory $\mathrm{T}$ cells are enriched in inflamed AD skin [7], favor a tissue phenotype and are distinct from freshly isolated CD4+ T cells.

Duhen et al. [2] identified Th22 cells as a distinct subset of CD4+ effector T cells. Moreover, they showed that 5 days

coccal exotoxins (SEB and $\alpha$-toxin). Cells were either left unstimulated (medium control) or were stimulated for 72 and $144 \mathrm{~h}$ with $\alpha$-toxin or SEB as indicated. Cell-free culture supernatants were quantified for IL-22 secretion by ELISA. ${ }^{*} \mathrm{p}<0.05$; ${ }^{* *} \mathrm{p}<0.01$; $* * * \mathrm{p}<0.001$.

of cell culture of human naïve CD4+ T cells with anti-CD3/ anti-CD28 in the presence of IL- 6 and TNF- $\alpha$ boosted the differentiation of Th22 cells and concluded that IL- 6 and TNF- $\alpha$ enhance Th22 differentiation. Beside IL-22, IL-17 and IFN- $\gamma$ were investigated as putative coproducing cyto- 
kines $[2,8]$. We could show that 3 days of cell culture of human memory T cells with IL-2 and anti-CD3/anti-CD28 could induce more IL-22 secretion compared to freshly isolated memory T cells and that adding IL- 6 and TNF- $\alpha$ to the cell culture could further enhance this effect, as previously shown by Duhen et al. [2] for naïve T cells. We could further reveal that 6 days of cell culture enhanced IL-22 secretion in human memory T cells with and without IL-2 and anti-CD3/anti-CD28 as well as in T cells stimulated with IL- 6 and TNF- $\alpha$. However, in contrast to Duhen et al., Th22-promoting conditions with IL- 6 and TNF- $\alpha$ failed to further enhance IL-22 secretion in human memory T cells after 6 days of cell culture compared to memory T cells generated with IL-2 and anti-CD3/anti-CD28 (table 1b). It is tempting to hypothesize whether this is due to T cell overstimulation with consecutive exhaustion. We could confirm that under Th22-promoting conditions with IL-6 and TNF- $\alpha$, memory Th2 2 cells secreted only low amounts of IFN- $\gamma$ and IL-17 [2]. However, memory Th2 2 cells coproduced high amounts of IL-13 in our hands and are therefore considered as Th22/Th2 cells, whereas this cytokine was not investigated by Duhen et al. in naïve T cells. Trifari et al. [1] described a population of CCR4+CCR6+CCR10+ human memory $\mathrm{T}$ cells that produced IL-22 and IL-13 but not IFN- $\gamma$ or IL-17 and called them Th22 cells, which is in line with our data.

Here we could show that SEB and $a$-toxin strongly induced IL-22 secretion in freshly isolated peripheral blood memory $\mathrm{T}$ cells as well as in Th22 cells derived from memory $\mathrm{T}$ cells in long-term cell culture without polarization and Th22 cells generated under Th22-promoting conditions with IL- 6 and TNF- $\alpha$. Although IL-22 secretion was higher in Th22 cells compared to freshly isolated memory T cells (online suppl. fig. S1), IL-22 induction by staphylococcal exotoxins did not differ between these three cell types (fig. $2 a-c)$. This implicates the following: (1) in vivo existing memory T cells are able to produce similar amounts of IL-22 upon staphylococcal exotoxin stimulation compared to in vitro highly polarized Th22 cells and (2) skin-infiltrating memory T cells can produce IL-22 rapidly upon contact with staphylococcal exotoxins.

Interestingly, $\alpha$-toxin stimulation increased IL-22 secretion of PBMCs and CD4+ T cells obtained from AD and psoriasis patients and healthy controls in our previously published work [4]. We could not detect any differences in staphylococcal exotoxin-induced IL-22 secretion between blood-derived Th22 cells from AD patients and cells from psoriasis patients and healthy controls in the present study. It is tempting to hypothesize whether longterm $\mathrm{T}$ cell culture that is necessary for Th22 polarization can mask inflammatory in vivo conditions and whether it is rather artificial than suitable for investigating chronic inflammatory skin diseases such as $\mathrm{AD}$ and psoriasis. The role of Th22 cells, including related cytokines and transcription factors, in the context of staphylococcal colonization and infection in $\mathrm{AD}$ should be further investigated directly in the skin compartment to elucidate its role in the pathogenesis and maintenance of eczema.

\section{Acknowledgment}

We would like to thank Mrs. Kathrin Baumert for her excellent technical assistance. This study was supported by the 'Hochschulinterne Leistungsfoerderung (HiLF)' program of Hannover Medical School and Deutsche Forschungsgemeinschaft (Research Training Group: GRK 1441/1).

\section{Disclosure Statement}

The authors have no financial conflicts of interest to declare.

\section{References}

$>1$ Trifari S, Kaplan CD, Tran EH, Crellin NK, Spits H: Identification of a human helper T cell population that has abundant production of interleukin 22 and is distinct from Th-17, Th1 and Th2 cells. Nat Immunol 2009;10:864-871.

$>2$ Duhen T, Geiger R, Jarrossay D, Lanzavecchia A, Sallusto F: Production of interleukin 22 but not interleukin 17 by a subset of human skinhoming memory T cells. Nat Immunol 2009; 10:857-863.

-3 Auriemma M, Vianale G, Amerio P, Reale M: Cytokines and T cells in atopic dermatitis. Eur Cytokine Netw 2013;24:37-44.
4 Niebuhr M, Scharonow H, Gathmann M Mamerow D, Werfel T: Staphylococcal exotoxins are strong inducers of Interleukin (IL)22: a potential role for atopic dermatitis. J Allergy Clin Immunol 2010;126:1176-1183.

$>5$ Niebuhr M, Langnickel J, Begemann G, Baumert K, Draing C, Werfel T: Dysregulation of TLR-2 induced effects in monocytes from patients with atopic dermatitis: impact of the TLR-2 R753Q polymorphism. Allergy 2008; 63:728-734.
6 Hanifin JM, Rajka G: Diagnostic features of atopic dermatitis. Acta Derm Venereol Suppl (Stockh) 1980;92:44-47.

7 Werfel T: The role of leukocytes, keratinocytes, and allergen-specific IgE in the development of atopic dermatitis. J Invest Dermatol 2009;129:1878-1891.

$>8$ Hijnen D, Knol EF, Gent YY, Giovannone B, Beijn SJ, Kupper TS, Bruijnzeel-Koomen CA, Clark RA: CD8+ T cells in the lesional skin of atopic dermatitis and psoriasis patients are an important source of IFN- $\gamma$, IL-13, IL-17 and IL-22. J Invest Dermatol 2013;133:973-979.
IL-22 Induction in Th22 Cells by

Staphylococcal Exotoxins
Int Arch Allergy Immunol 2014;165:35-39 DOI: $10.1159 / 000367923$ 